\title{
Mining Permission Patterns for Contrasting Clean and Malicious Android Applications
}

\author{
Veelasha Moonsamy, Jia Rong, Shaowu Liu \\ School of Information Technology, Deakin University, 221 Burwood Highway, Vic 3125, \\ Australia.
}

\begin{abstract}
Android application uses permission system to regulate the access to system resources and users' privacy-relevant information. Existing work have demonstrated several techniques to study the required permissions declared by the developers, but few attention has been paid for used permissions. Besides, no specific permission combination is identified to be effective for malware detection. To fill these gaps, we have proposed a novel pattern mining algorithm to identify a set of contrast permission patterns that aim to detect the difference between clean and malicious applications. In addition, we used a benchmark malware dataset and collected a set of 1227 clean applications to evaluate the performance of the proposed algorithm. Valuable findings are obtained by analyzing the returned contrast permission patterns.
\end{abstract}

Keywords: Android Permission, Data Mining, Biclustering, Contrast Mining, Permission Pattern

\section{Introduction}

Between 2010 and 2013, the global telephony industry has witnessed an upsurge in the sales of smartphones. Smartphone is a term used to describe a mobile device equipped with enhanced computing capability and connectivity [1], such as Nexus by Google [2], iPhone by Apple [3, Blackberry by RIM [4] and Windows Phone by Microsoft [5]. A smartphone is usually sold with an in-built Operating System (OS) together with a number of applications pre-installed by the device manufacturer. Application, the equivalent

Email addresses: v.moonsamy@research.deakin.edu.au (Veelasha Moonsamy), jiarong@acm.org (Jia Rong), swliu@deakin.edu.au (Shaowu Liu) 
of software on the PC platform, enhances the smartphone's functionality and supports interactions with the user to accomplish a set of tasks. Calendar, address book, alarm clock, media player and web browser are the common applications provided by the device manufacturers. More importantly, there exists an application, commonly known as the "application store", on every smartphone that provides the smartphone user access to online application markets in order to search and download additional applications on their smartphones.

Every device manufacturer hosts an application market for its own smartphone OS, such as Apple's App Store [6], Google's Google Play [8], Blackberry's App World [7]. This type of application market is often referred to as the official application market. However, the competitive nature and regulations imposed by official application market have led to a surge in the number of independent application markets, also known as third-party application markets. Third-party application markets are till date a popular choice amongst smartphone users because of the availability of a large variety of free/low-priced applications and the inexistence of application vetting process. Nonetheless, one cannot guarantee the cleanliness of such applications [9].

To effectively detect malicious applications (also known as malware), many efforts have been contributed, in the last five years, into studying the nature of smartphone platforms and their applications. In addition to being the leading smartphone platform, Android is also the most infected OS due to an exponential growth in the number of malware deployed on the application markets. As one of its security measures, the Android platform employs a permission system to restrict applications' privileges in order to secure user privacy-relevant resources [12]. An application needs to get the user's approval for the requested permissions to access these restricted resources. Thus, the permission system was designed to protect a user from applications with invasive behaviors, but its effectiveness highly depends on the user's comprehension of permission approval. We refer to the permissions that are requested during application installation as required permissions.

Unfortunately, not all the users read or understand the warnings of required permissions shown during application installation. To improve this situation, many researchers have tried to interpret Android permissions and their combinations [13-16]. Frank et al. [12] proposed a probability model to identify the common required permission patterns for all Android applications. Zhou and Jiang [11] listed the top required permissions for both clean and malicious applications, but only individual permissions were considered by frequency counting. The problem of identifying patterns in permission 
combinations that can provide better performance for malware detection still remains.

Furthermore, in the existing literature, only required permissions have been considered in permission pattern mining and no work has incorporated used permissions, which can be described as follows: Whenever an Application Programming Interface (API) call is invoked during the execution of an application, the smartphone OS will verify if the API call is permissionprotected before proceeding to execute the call; such permissions are referred to as used permissions. To our knowledge, we are the first to consider both the required and used permissions that are extracted from static analysis by the Andrubis system (http://anubis.iseclab.org) [17]. Accordingly, our aim is to propose an efficient pattern mining method to identify a set of contrast permission patterns that effectively distinguish malware from the clean applications.

In order to use pattern mining technique to identify the desired permission patterns, we utilized a clean dataset and a malicious one. In 2012, Zhou and Jiang [11] published the first benchmark dataset of malicious applications in 49 malware families and was collected from third-party markets between August 2010 to October 2011. This is an ideal malware dataset for our experiment as it includes an extensive list of malware families collected over 14 months. On the other hand, due to the unavailability of a clean dataset published during the same time period as Zhou and Jiang's, we collected our own set of clean applications. Those clean applications were downloaded from two popular third-party Android applications markets: SlideME (http://slideme.org) and Pandaapp (http://android.pandaapp.com). We sorted the clean applications based on their upload dates and the ratings given by the users, and only the top ones were selected. Each application was scanned by forty-three antivirus engines on VirusTotal [18], and only those that passed all virus tests were considered as "clean" and included in our clean dataset. Similar to Zhou and Jiang, we represent applications in the collected clean dataset using a vector of 130 binary values, each of which is associated with one of the 130 official Android permissions. A value 1 is assigned to a permission only if it is required or used by an application, otherwise, 0 is given instead.

The novelty and contributions of this work can be summarized as follows:

- We proposed a Contrast Permission Pattern Mining algorithm to identify the interesting permission sets as the patterns can be used to distinguish malicious applications from clean ones.

- Beyond the current studies that focused on required permissions only, 
we also considered the used permissions and our experimental work showed that both required and used permissions were important to be considered in late malware detection task.

- We collected a new dataset that contains 1227 clean applications that were uploaded to third-party markets from August 2010 to October 2011.

- We utilized a hierarchical Biclustering method to initially analyze both clean and malware datasets. The resulting figures provided a straightforward visualization of the data distribution, from which we built up our model of mining a set of permissions rather than using individual permissions as the patterns.

The rest of the paper is organized as follows: Section 2 briefly reviews the concepts of the Android platform, its applications, the permission system and the current research work in malware detection. In Section 3 , we present our initial analysis on the collected datasets using statistical method and biclustering followed by our proposed contrast pattern mining algorithm. The experiments and the empirical results are then reported in Section 4 followed by a further discussion on our findings. Finally, Section 5 concludes the entire paper together with our future work.

\section{Background and Related Work}

\subsection{Android}

Android is a Linux-based OS, which was designed and developed by the Open Handset Alliance in 2007 [19]. The Android platform is made up of multiple layers consisting of the OS, Java libraries and basic built-in applications [20]. Additional applications can be downloaded and installed from either official or third-party markets.

Google provides the application developer community with a Software Development Kit (SDK) [21] to build Android applications. The SDK includes a collection of Java libraries and classes, sample applications and developer documentations. The SDK can be used as a plug-in for Eclipse IDE [22] and therefore allows developers to code their applications in a rich Java environment. One particularly useful feature of the SDK is the $A n$ droid emulator which allows developers to test their applications in virtual devices supporting various versions of Android.

An Android application includes two folders and one file: (i) Class, (ii) Resources and (iii) AndroidManifest.xml. The Class folder contains the 
application's source code in Java; the Resources folder stores the multimedia files; and the AndroidManifest.xml file lists the required permissions that are declared by the developer. When the Java source code is ready, it is then compiled and converted into Dalvik byte code [23] and bundled with the Resources folder and AndroidManifest.xml file to generate the Android Application Package (APK). Finally, before the APK can be installed on a device or emulator, the developer has to generate a key and sign the application.

Android developers can upload their applications to either the official market, Google Play [24, or any third-party market. To secure the privacyrelevant resources for its users, Google provides automatic antivirus scanning. The applications will be rejected from Google Play if any malicious content is detected. From 2012, Google has extended its antivirus service on the new Android 4.2 OS, which is claimed to be able to scan applications before they are installed on the device [26].

\subsubsection{Android Permission System}

Google applies the permission system as a measure to restrict access to privileged system resources. Application developers have to explicitly mention the permissions that need user's approval in the AndroidManifest.xml file. Android adopts an 'all-or-nothing' permission granting policy. Hence, the application is installed successfully only when the user chooses to grant access to all of the required permissions.

There are currently 130 official Android permissions and are categorized into four types: Normal, Dangerous, Signature and SignatureOrSystem [27]. Normal permissions do not require the user's approval but they can be viewed after the application has been installed. Dangerous permissions require the user's confirmation before the installation process starts; these permissions have access to restricted resources and can have a negative impact if used incorrectly. A permission in Signature category is granted without the user's knowledge only if the application is signed with the device manufacturer's certificate. The SignatureOrSystem permissions are granted only to the applications that are in the Android system image or are signed with the device manufacturer's certificate. Such permissions are used for special situations where the applications, built by multiple vendors, are stored in one system image and share specific features.

After an application is installed, a set of APIs will be called during the runtime. Each API call is associated with a particular permission. When an API call is made, the Android OS checks whether or not its associated

permission has been approved by the user. Only a matching result will 
lead to the execution of the API call. In this way, the required permissions are able to protect the user's private information from unauthorized access. However, an API call invocation cannot fully stop the malware developers from declaring required permissions for their applications. Based on the above observation, several studies have tried to identify the common required permissions that are frequently declared by Android application developers.

By applying the Self-Organizing Map (SOM) algorithm, Barrera et al. [13] studied the trends of permission requests from a dataset of 1,100 applications downloaded from the official market. Frank et al. [12] selected 188, 389 applications from the official market and analyzed the combinations of permissions requests by these applications. A probabilistic method was proposed to deduce the popular permission patterns based on the applications' popularity (ratings together with number of reviews), that is, the deviation of permission requests for high- and low-ranked applications. Bartel et al. 28] proposed an automated tool that can statistically analyze the methods defined in an application and subsequently generate the permissions required by the application. This in turn ensures that the user does not grant access to unnecessary permissions when installing the application. A model designed by Sanz et al. 29] is based on features which comprised solely of Android permissions.

The aforementioned existing work studied the applications that were collected mainly from the official market. The results and findings help us to understand the Android permission system and the patterns for normal permission requests. However, compared to clean applications, we are more interested in the unusual permission requests, which are considered as more valuable for the detection of Android malware.

\subsection{Android Malware Detection with Permissions}

Malware detection within the Android platform is gaining a fair amount of attention from researchers in both academia and industry; however, there is a lack of work on the detection of Android malware using permission patterns. Rassameeroj and Tanahashi [30] used visualization techniques and clustering algorithms to reveal normal and abnormal permission request combinations. They evaluated their methodology on a dataset comprising of 999 applications. After analyzing the extracted permission combinations, they claimed that nearly $8 \%$ of the applications were potential malicious.

Chia et al. [16] argued that the current user-rating system was not a reliable source of measurement to predict whether or not an application was malicious. Their dataset consisted of 650 applications from the official market and 1,210 applications from a third-party market. The required permissions 
were extracted from the dataset, together with other application-related information to develop a risk signal mechanism for detecting malware.

Sahs and Khan [31] focused on feature representation as one of the challenges to malware detection. The features to be represented included: (i) permissions extracted from manifest files and (ii) control flow graphs for each method in an application. Each feature was processed independently using multiple kernels and applied a one-class Support Vector Machine to train the classifiers. However, the evaluation results showed that the common features existing in both clean and malware datasets cause detection error rate.

$\mathrm{Wu}$ et al. 32 put forward a static feature-based technique that can aid towards malware detection. First, they apply K-means algorithms to generate the clusters and use Singular Value Decomposition to determine the number of clusters. In the second step, they classify clean and malicious applications using the $k$-Nearest Neighbor (kNN) algorithm.

Zhou et al. 33. proposed a two-layered system known as, DroidRanger and makes use of "permission-based behavioral foot-printing and heuristicsbased filtering". The authors observed that the permissions extracted from the AndroidManifest.xml files of malicious applications gave an insight into uncommon permission requests by some malware families. In Sanz et al.'s work [29], they extracted the permissions and the hardware features to build the feature set. As a result, clean applications require two to three permissions on average, but some of malicious applications only have one permission and are still able to carry out the attack.

Most of the work extracted a feature set to represent the applications. The information carried by those features were different from work to work. There is no evidence to show which features give the best detection result; nonetheless, each study considered only the required permissions. Accordingly, we consider taking the permissions as the only features to represent the applications and expect to find specific permission patterns to show the difference between clean and malicious applications.

\subsection{Summary}

Malware proliferation is rising exponentially and the attack vectors used by malware authors are getting more sophisticated. Current solutions proposed to thwart attacks by malicious applications will struggle to keep up with the increase of malware. The Android platform relies heavily on its permission system to control access to restricted system resources and private information stored on the smartphone. As demonstrated by [12], [34], 
and [35], permissions that are requested by applications during installation can be helpful in identifying permission patterns.

However, we identify the following problems in the existing literature:

Problem 1 What required permission patterns can be used to detect malicious applications?

Problem 2 What used permission patterns can be used to detect malicious applications?

Problem 3 Can we extract useful information by incorporating used permissions into the permission patterns?

Problem 4 What method can we use to identify these expected permission patterns?

In this paper, we aim to extend the current statistical method used for identifying permission patterns in Android applications by applying pattern mining techniques to a set of clean and malicious applications in order to better understand the similarities and differences between these two datasets. With the help of visualization graphs, we establish possible connections between required permissions and used permissions in order to extrapolate emerging permission patterns that are frequently requested by applications. Then, we apply contrast set mining on the permissions patterns from clean and malicious applications to identify which patterns are most prevalent in each dataset.

\section{Mining Permission Patterns}

The most common method used to analyze Android permissions are statistical-based; for instance, frequency counting by Zhou and Jiang [11] and probabilistic model by Frank et al. [36]. Thus, we started our work by performing an initial analysis on the clean and malware datasets using frequency counting and extended it to incorporate used permissions. To further extend the work of Barrera et al. [13] who utilized SOM for application clustering and visualization, we applied the biclustering algorithm to not only group the applications but also their respective permissions. Finally, a novel contrast permission pattern mining algorithm is presented to identify specific permission patterns that can help distinguish between clean and malicious applications. 


\subsection{Classic Statistical Analysis on Android Permissions}

We performed a statistical analysis to study both the required and used permissions for clean and malicious applications. Hence, the following four sub-datasets were extracted: (1) Required permissions for clean applications; (2) Required permissions for malicious applications; (3) Used permissions for clean applications; and (4) Used permissions for malicious applications. Direct frequency counting is employed on all four sub-datasets to find out the most popular permissions required or used by clean and malicious applications.

Table 1: Top 20 Required Permissions by Clean and Malicious Applications

\begin{tabular}{l|l|l|l}
\hline \multicolumn{2}{c}{ Clean Applications } & \multicolumn{2}{c}{ Malicious Applications } \\
Required Permission & Frequency & Required Permission & Frequency \\
\hline INTERNET & $1121(91.36 \%)$ & INTERNET & $1199(97.72 \%)$ \\
ACCESS_NETWORK_STATE & $663(54.03 \%)$ & ACCESS_COARSE_LOCATION & $1146(93.40 \%)$ \\
READ_PHONE_STATE & $391(31.87 \%)$ & VIBRATE & $994(81.01 \%)$ \\
WRITE_EXTERNAL_STORAGE & $362(29.50 \%)$ & WRITE_EXTERNAL_STORAGE & $823(67.07 \%)$ \\
ACCESS_COARSE_LOCATION & $236(19.23 \%)$ & READ_SMS & $779(63.49 \%)$ \\
VIBRATE & $210(17.11 \%)$ & WRITE_SMS & $762(62.10 \%)$ \\
WAKE_LOCK & $188(15.32 \%)$ & READ_CONTACTS & $680(55.42 \%)$ \\
ACCESS_FINE_LOCATION & $162(13.20 \%)$ & BLUETOOTH & $633(51.59 \%)$ \\
GET_TASKS & $125(10.19 \%)$ & WRITE_CONTACTS & $542(44.17 \%)$ \\
SET_WALLPAPER & $102(8.31 \%)$ & DISABLE_KEYGUARD & $491(40.02 \%)$ \\
ACCESS_WIFI_STATE & $64(5.22 \%)$ & WAKE_LOCK & $471(38.39 \%)$ \\
RECEIVE_BOOT_COMPLETED & $60(4.89 \%)$ & RECORD_AUDIO & $461(37.57 \%)$ \\
READ_CONTACTS & $58(4.73 \%)$ & ACCESS_FINE_LOCATION & $446(36.35 \%)$ \\
WRITE_SETTINGS & $45(3.67 \%)$ & ACCESS_NETWORK_STATE & $416(33.90 \%)$ \\
CAMERA & $43(3.50 \%)$ & READ_PHONE_STATE & $414(33.74 \%)$ \\
CALL_PHONE & $42(3.42 \%)$ & SET_ORIENTATION & $413(33.66 \%)$ \\
SEND_SMS & $34(2.77 \%)$ & CHANGE_WIFI_STATE & $384(31.30 \%)$ \\
RESTART_PACKAGES & $32(2.61 \%)$ & READ_LOGS & $361(29.42 \%)$ \\
RECEIVE_SMS & $31(2.53 \%)$ & BLUETOOTH_ADMIN & $342(27.87 \%)$ \\
RECORD_AUDIO & $27(2.20 \%)$ & RECEIVE_BOOT_COMPLETED & $325(26.49 \%)$ \\
\hline
\end{tabular}

After comparing the top 20 required permissions for clean and malicious applications listed in Table 1, we found that the malicious applications requested a total of 14,758 permissions, which was much more than clean applications (4, 470 permissions). Among these permissions, we found some of them only appeared in one dataset, that is, those permissions are only requested or used by clean applications but not malicious ones, and vice versa. We call these permissions as 'unique permissions'. Similarly, we name those permissions that appear in both clean and malware datasets as 'common permissions'. Totally, there are 33 unique required permissions for clean applications and 20 for malicious ones; and also 70 common required permissions. Another 5 permissions have never been requested by any application. For used permissions, 9 unique ones for clean applications and only 4 for malicious ones. The number of common used permissions dropped to 28 , and a large number of 87 permissions have never been used by any application. Four common permissions that were most frequently 
required by both clean and malicious applications are as follows: INTERNET, ACCESS_COARSE_LOCATION, WRITE_EXTERNAL_STORAGE and VIBRATE. In contrast, there were nine out of twenty required permissions appeared frequently in malware dataset than in clean one. Moreover, when comparing the top 20 used permissions in clean and malicious applications in Table 2, we observed that sixteen out of twenty popular used permissions are common in both datasets.

Table 2: Top 20 Used Permissions by Clean and Malicious Applications

\begin{tabular}{l|l|l|l}
\hline \multicolumn{2}{c}{ Clean Applications } & \multicolumn{2}{c}{ Malicious Applications } \\
Used Permission & Frequency & Used Permission & Frequency \\
\hline INTERNET & $1029(83.86 \%)$ & INTERNET & $1161(94.62 \%)$ \\
WAKE_LOCK & $816(66.50 \%)$ & ACCESS_COARSE_LOCATION & $1125(91.69 \%)$ \\
ACCESS_NETWORK_STATE & $738(60.15 \%)$ & VIBRATE & $954(77.75 \%)$ \\
VIBRATE & $608(49.55 \%)$ & WAKE_LOCK & $826(67.32 \%)$ \\
READ_PHONE_STATE & $457(37.25 \%)$ & ACCESS_WIFI_STATE & $584(47.60 \%)$ \\
ACCESS_COARSE_LOCATION & $372(30.32 \%)$ & ACCESS_NETWORK_STATE & $519(42.30 \%)$ \\
SET_WALLPAPER & $126(10.27 \%)$ & READ_SMS & $473(38.55 \%)$ \\
ACCESS_FINE_LOCATION & $116(9.45 \%)$ & WRITE_CONTACTS & $426(34.72 \%)$ \\
GET_ACCOUNTS & $98(7.99 \%)$ & READ_PHONE_STATE & $354(28.85 \%)$ \\
ACCESS_WIFI_STATE & $85(6.93 \%)$ & RECORD_AUDIO & $319(26.00 \%)$ \\
READ_SMS & $82(6.68 \%)$ & SET_WALLPAPER & $297(24.21 \%)$ \\
RESTART_PACKAGES & $65(5.30 \%)$ & ACCESS_FINE_LOCATION & $199(16.22 \%)$ \\
GET_TASKS & $61(4.97 \%)$ & GET_ACCOUNTS & $178(14.51 \%)$ \\
CHANGE_CONFIGURATION & $55(4.48 \%)$ & GET_TASKS & $124(10.11 \%)$ \\
RECEIVE_SMS & $37(3.02 \%)$ & RECEIVE_BOOT_COMPLETED & $111(9.05 \%)$ \\
FLASHLIGHT & $37(3.02 \%)$ & ACCESS_CACHE_FILESYSTEM & $101(8.23 \%)$ \\
WRITE_CONTACTS & $34(2.77 \%)$ & WRTIE_OWNER_DATA & $59(4.81 \%)$ \\
RECEIVE_BOOT_COMPLETED & $23(1.87 \%)$ & CHANGE_CONFIGURATION & $52(4.24 \%)$ \\
WRTIE_OWNER_DATA & $12(0.98 \%)$ & READ_HISTORY_BOOKMARKS & $49(3.99 \%)$ \\
WRITE_SETTINGS & $10(0.81 \%)$ & EXPAND_STATUS_BAR & $41(3.34 \%)$ \\
\hline
\end{tabular}

Statistical method such as direct frequency counting is suitable for identifying single permissions that are popular in each sub-datasets respectively. However, it still requires further manual checking to confirm the obtained permission lists for clean and malicious applications. This, in turn, further complicates the counting process if permission combinations are to be considered instead of individual permissions. Therefore, we continued our analysis of Android permissions by applying the biclustering algorithm as a next step in order to provide visualization of the relationship between permissions and applications.

\subsection{Visualization Using Biclustering}

Biclustering [37] is a special cluster analysis method, which applies classic clustering to both rows and columns, simultaneously, in a two-dimensional data matrix. In this work, biclustering can help group applications that request or use different permission combinations as well as show us the clusters of permission combinations based on the various applications associated with them. 
The biclustering is achieved by performing Agglomerative Hierarchical Clustering (AHC) [38, 39] on both dimensions of the data matrix. The $A H C$ is first applied along the columns of the data matrix and then applied along the rows of the row-clustered data matrix.

Unlike the common clustering methods which identify a single set of clusters, the $A H C$ is a bottom-up clustering method that seeks to identify clusters with sub-clusters. It forms a multilevel hierarchical clustering tree, where lower-level clusters are joined to form higher-level clusters. The steps can be described as follows:

Step 1: Generate the Disjoint Clusters - The $A H C$ starts with every single data object, i.e. each data object is assigned to a separate cluster.

Step 2: Form a Distance Matrix - The pairwise distances between the disjoint clusters are calculated using the Ward's linkage [40] and are used to initialize the distance matrix.

Step 3: Merge two Closest Clusters - Based on the distance matrix, each cluster is merged with the next closest cluster with the shortest distance.

Step 4: Update the Distance Matrix - After the merging, the distance matrix needs to be updated by calculating the new distance between each pair of merged clusters.

Step 5: Obtain the Hierarchical Clustering Tree - Steps 3 and 4 are repeated until all clusters are merged into one large single cluster, resulting in a complete hierarchical clustering tree.

We applied the above biclustering steps on two separate sub-datasets extracted from the malware dataset and our collected clean one. One subdataset contains all clean and malicious applications with required permissions (see Fig. 1a); the second sub-dataset includes the same applications but with used permissions (see Fig. 1b).

As a result, we had two output matrices - the required permissions are shown in Fig. 2 and the used permissions are in Fig. 3. Based on the statistical analysis presented in Section 3.1, there exist unique and common permissions for either clean or malicious applications. As binary values are shown in these matrices, we manually picked up four colors to mark the values to visualize different types of permissions for clean and malicious applications:

- Dark Green shows Common permissions for clean applications; 


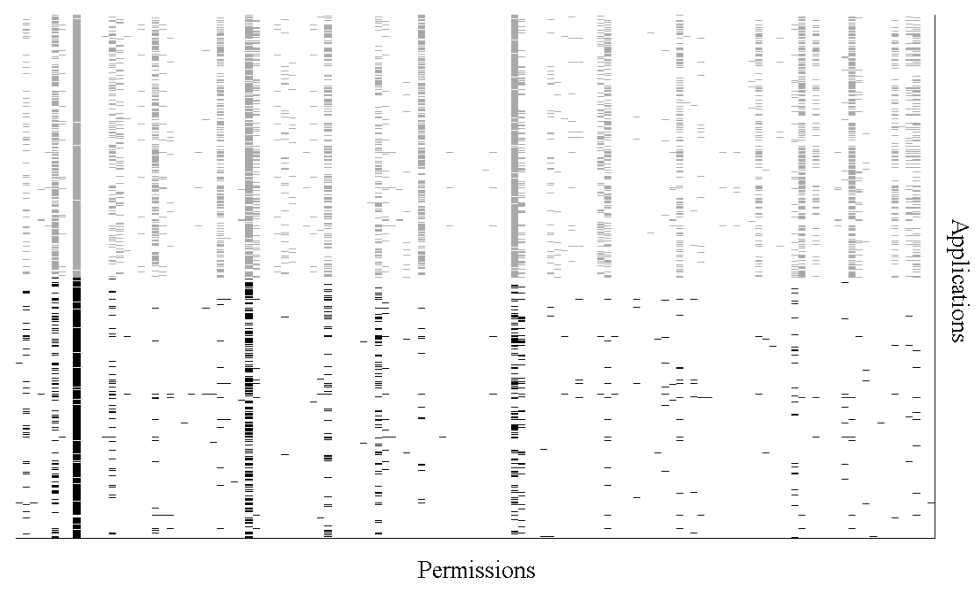

(a) Required Permissions

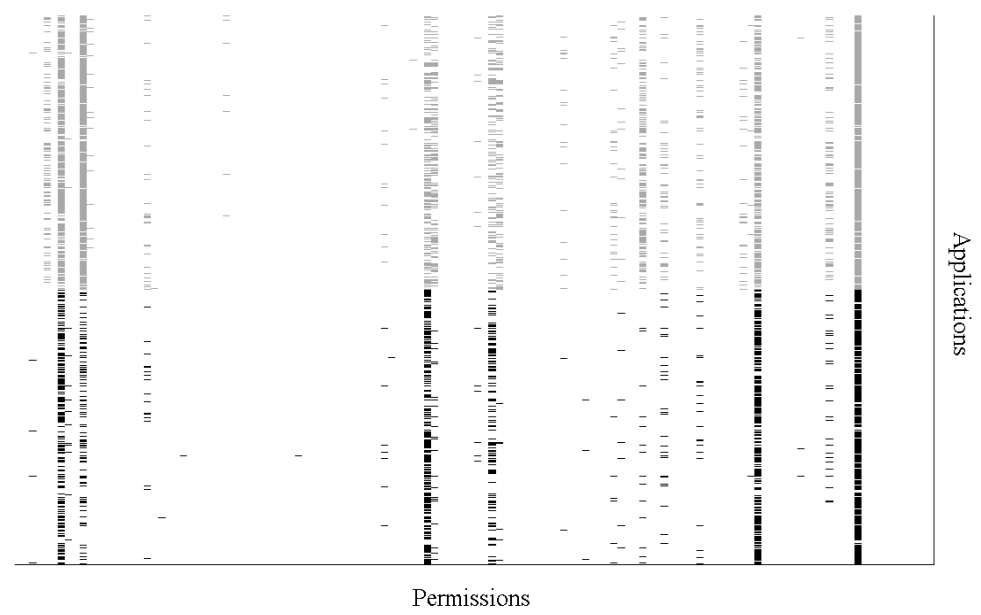

(b) Used Permissions

Figure 1: Visualization of Sub-datasets for Biclustering: white for 0s; gray for 1s in clean applications; and black for 1s in malicious applications 
- Sandy Brown shows Common permissions for malicious applications;

- Blue indicates Unique permissions for clean applications; and

- Red indicates Unique permissions for malicious applications.

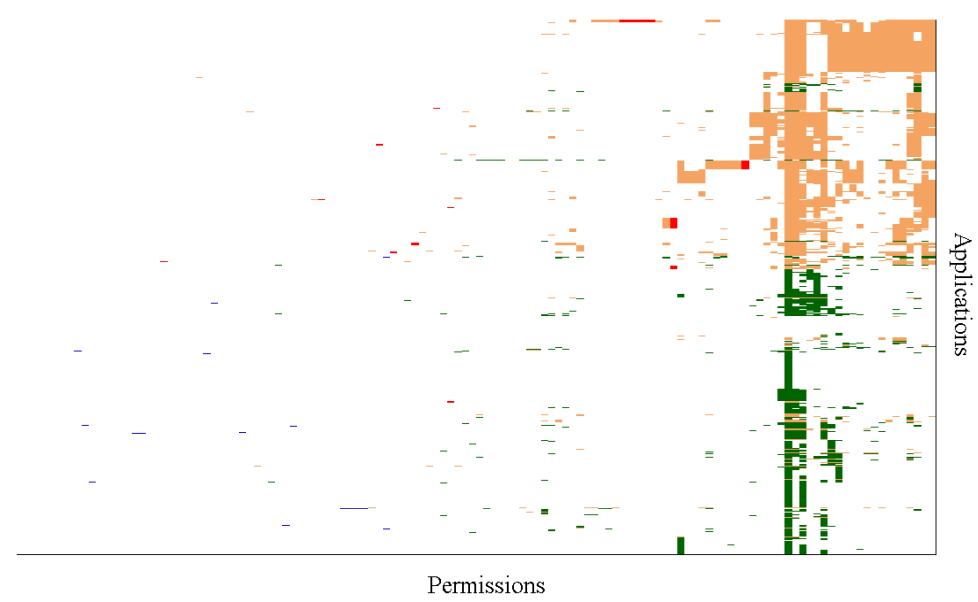

Figure 2: Resulting Matrix for Required Permissions by Biclustering

From these two figures, we can easily observe that more permissions are declared as required than those that are actually used. This further confirms our results from the statistical analysis in Section 3.1. Furthermore, generally, malicious applications either request or use more permissions than clean applications. In normal cases, the unique permissions should perform better than the common permissions to contrast between clean and malicious applications. However, from the resulting matrices, we only have few unique permissions shown in bright red and blue colors compared to the large set of common permissions. Therefore, when applying only statistical methods, it is easy to ignore those unique permissions because of their low frequency. Furthermore, it is obvious to see the color blocks in both figures, which indicate specific permission combinations have potential capability to group applications in separate clusters. The aforementioned observations give rise to the following challenges, which are addressed in the rest of the paper: How can we use the unique permissions for contrast detection? How can we find out the permission combinations and use them as the patterns for malware detection? 


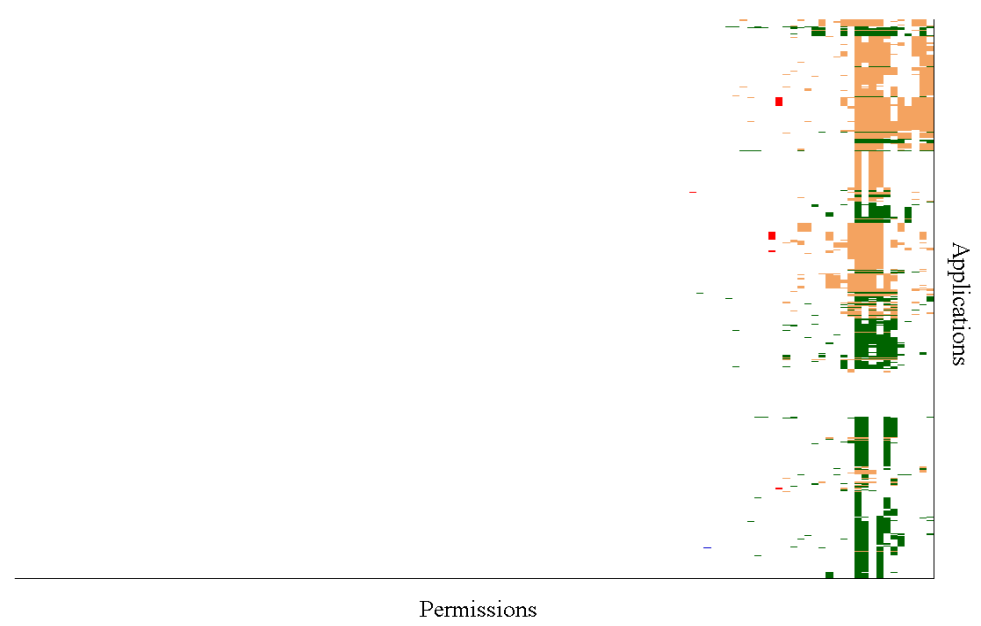

Figure 3: Resulting Matrix for Used Permissions by Biclustering

\subsection{Contrast Permission Pattern Mining}

In order to tackle the two challenges presented in Section 3.2 , the Contrast Permission Pattern Mining (CPPM) is proposed. The output permission patterns are expected to have the ability to indicate the difference between the clean and malicious applications. CPPM was designed to process more than one dataset and take both common and unique permissions and their combinations into consideration. Two major processes are involved in CPPM: (1) candidate permission itemset generation, and (2) contrast permission pattern selection.

\subsubsection{Candidate Permission Itemset Generation}

The purpose of this process is to obtain a number of candidate permission combinations that are most likely to be the expected contrasting patterns. $C P P M$ takes at least two datasets as input. In this case two datasets are loaded, each of which contains either clean or malicious applications. We generate the candidate permission itemsets for every dataset using the same procedure, which can be described as the following two steps:

\section{Apriori-based Itemset Enumeration}

Given $D x$ is one of the input datasets with either required or used permissions, which contains $n$ Android applications. Let $I=\{A, B, C \ldots\}$ be the set of possible items (or permissions requested or used by the 
applications) in Dx. The Apriori-based approach [41, enumerates candidate itemset from the simplest structure with only a single item. Based on this single item, a more complex itemset is then obtained by adding new items. This joining operation is repeated continuously to increase the number of the items in the itemsets. In each iteration, only one item is added into the existing candidate itemset. One item will not appear twice in one itemset. However, the Apriori-based approach can generate a large number of candidate itemsets with high computational cost. To solve this problem, we employ a supportbased pruning technique to reduce the number of candidate itemsets and consequently, the experimental time.

\section{Support-based Candidate Pruning}

Support is usually used to measure the occurrence frequency of a certain item or itemset in a dataset. Let $A, B \subseteq I$ be two items, and $\{A, B\}$ forms a candidate itemset. The support of the candidate itemset $\{A, B\}$ can be calculated by:

$$
\operatorname{supp}(A, B)=\frac{\text { number of applications that contain } A \text { and } B \text { in } D x}{\text { total number of applications in } D x}
$$

The candidate itemset $\{A, B\}$ is considered as frequent only if $\operatorname{supp}(A$, $B) \geq \delta_{\text {supp }}$, where $\delta_{\text {supp }}$ is user-specified minimum support threshold. In classic pattern mining methods, only the frequent itemset is considered. Any itemset with a lower support than the pre-determined threshold is treated as infrequent and discarded immediately. However, in our case, both the statistical analysis and biclustering results show most of the unique permissions are requested or used by few applications. This is an indication that their support values are definitely low. In order to inadvertently miss any valuable patterns, we decide to take both frequent and infrequent candidate itemsets, but only use frequent ones to generate new candidate itemsets to cut down the computational cost.

\subsubsection{Contrast Permission Pattern Selection}

The permission itemsets generated from Section 3.3.1 need to be reduced according to the pre-defined selection criteria. This process guarantees that the output itemsets are highly contrasted between clean and malicious applications. The contrasting permission patterns reflect the different behaviors present amongst the applications in the two datasets. If one permission 
itemset is frequent in one dataset, it is often considered to carry more common features than the infrequent ones. Therefore, the selection of specific contrast permission pattern is based on comparison of its support in both datasets. The bigger the difference in support values, the greater the contrast a permission pattern has.

Given one candidate permission itemset $\{A, B\}$ and its support values in clean and malware datasets, $\operatorname{supp}(A, B)_{\text {clean }}$ and $\operatorname{supp}(A, B)_{\text {malicious }}$, calculate the difference by $\operatorname{diff}(A, B)=\operatorname{supp}(A, B)_{\text {clean }}-\operatorname{supp}(A, B)_{\text {malicious }}$. $\{A, B\}$ is identified as a contrasted permission pattern only if $\operatorname{diff}(A, B) \geq$ $\delta_{\text {diff }}$, where $\delta_{\text {diff }}$ is a user-specified minimum support difference. All the candidate permission itemsets need to be tested using this approach, and the ones with big support difference will be selected as the final output contrast permission patterns.

\section{Experiments and Results}

\subsection{Experiment Settings}

According to the statistical analysis and biclustering resulting figures, not all the permissions are required or used. In the experiment to evaluate the proposed Contrast Permission Pattern Mining algorithm, we ignore the permissions that are not required or used in each sub-datasets respectively. Table 3 gives more details of the four new sub-datasets.

The statistical analysis results also show that only a small set of permissions have support that are greater than 0.1 (10\%), so we follow the previous studies [42 44] to set 0.05 as an acceptable value for minimum support threshold for all four sub-datasets in CPPM. The minimum support difference threshold is set to be $0.15(15 \%)$ and applied to filter out itemsets that are highly contrasted between clean and malicious applications.

Table 3: Four Sub-datasets Used in Contrast Permission Pattern Mining Experiments

\begin{tabular}{ll|c|c}
\hline & Dataset & Permission involved & Permission Discarded \\
\hline 1 & Clean_Required & 103 & 27 \\
2 & Malicious_Required & 90 & 40 \\
3 & Clean_Used & 37 & 93 \\
4 & Malicious_Used & 31 & 99 \\
\hline
\end{tabular}


Table 4: Permission Index

\begin{tabular}{c|c|l}
\hline Permission Category & Permission ID & Permission Name \\
\hline Normal & $p m s 0001$ & INTERNET \\
Normal & $p m s 0006$ & ACCESS_NETWORK_STATE \\
Normal & $p m s 0007$ & VIBRATE \\
Normal & $p m s 0012$ & RESTART_PACKAGES \\
Normal & $p m s 0013$ & RECEIVE_BOOT_COMPLETED \\
Normal & $p m s 0023$ & ACCESS_WIFI_STATE \\
\hline Dangerous & $p m s 0002$ & ACCESS_FINE_LOCATION \\
Dangerous & $p m s 0003$ & WAKE_LOCK \\
Dangerous & $p m s 0004$ & WRITE_EXTERNAL_STORAGE \\
Dangerous & $p m s 0005$ & READ_PHONE_STATE \\
Dangerous & $p m s 0008$ & READ_CONTACTS \\
Dangerous & $p m s 0011$ & READ_LOGS \\
Dangerous & $p m s 0020$ & ACCESS_COARSE_LOCATION \\
Dangerous & $p m s 0021$ & SEND_SMS \\
Dangerous & $p m s 0022$ & GET_TASKS \\
Dangerous & $p m s 0024$ & CHANGE_WIFI_STATE \\
Dangerous & $p m s 0028$ & WRITE_CONTACTS \\
Dangerous & $p m s 0029$ & RECEIVE_SMS \\
Dangerous & $p m s 0030$ & READ_SMS \\
Dangerous & $p m s 0031$ & WRITE_SMS \\
Dangerous & $p m s 0036$ & CALL_PHONE \\
\hline Signature & $p m s 0010$ & FACTORY_TEST \\
SignatureOrSystem & $p m s 0052$ & INSTALL_PACKAGES \\
\hline
\end{tabular}

\subsection{Contrast Permission Patterns}

Among the permission patterns that were generated, we found that 23 distinct permissions were present in the highly contrasted permission combinations as listed in Table 4. We classified the permissions based on the following permission categories: Normal, Dangerous, Signature and SignatureOrSystem. We recorded 6 permissions belonging to the Normal category, 15 permissions for the Dangerous category and 1 permission each for the Signature and SignatureOrSystem category.

We found that the generated permission combinations are correlated and differed between clean and malicious applications. Based on the experimental results, we recorded 56 required permission patterns that are unique to the malware dataset, 31 used permission patterns that only appear amongst malware, 17 required permission patterns and 9 used permission patterns that are present in both clean and malware dataset. These findings are formed as permission combinations which are listed in Table 5-13, and summarized with respect to the usage type of the permission in the following section. First, we define the four types of permission combinations as follows: 
(i) Unique Required Permission (URP): Required permission patterns present only in malware dataset

(ii) Unique Used Permission (UUP): Used permission patterns present only in malware dataset

(iii) Common Required Permission (CRP): Required permission patterns present in both clean and malware datasets

(iv) Common Used Permission (CUP): Used permission patterns present in both clean and malware datasets

\subsubsection{Unique Required Permission (URP) Patterns}

From Table 5 - 10, we present the permission patterns that were frequently required by the applications in our dataset. It is worth noted that these required permission patterns were unique to the malware dataset only; hence the support value for the clean applications is 0 .

Table 5: Unique Required Permission Sets in Malware Dataset (Normal Permissions)

\begin{tabular}{|c|c|c|c|}
\hline \multirow[t]{2}{*}{ Permission Set } & \multicolumn{2}{|c|}{ Support } & \multirow{2}{*}{$\begin{array}{l}\text { Permission Set } \\
\text { ID }\end{array}$} \\
\hline & Clean & Malware & \\
\hline pms0001, pms0005, pms0023 & 0 & 0.6309 & URPSet $_{1}$ \\
\hline pms $0001, p m s 0006, p m s 0023$ & 0 & 0.6031 & $\mathrm{URPSet}_{2}$ \\
\hline pms0001, pms 0013 & 0 & 0.5542 & $\mathrm{URPSet}_{3}$ \\
\hline pms 0006, pms 0013 & 0 & 0.5168 & $\mathrm{URPSet}_{4}$ \\
\hline pms0006, pms0031 & 0 & 0.4964 & URPSet $_{5}$ \\
\hline pms 0001, pms 0021 & 0 & 0.4312 & $\mathrm{URPSet}_{6}$ \\
\hline pms 0013, pms 0023 & 0 & 0.4263 & $\mathrm{URPSet}_{7}$ \\
\hline pms 0021, pms 0029 & 0 & 0.3701 & URPSet $_{8}$ \\
\hline pms 0004, pms 0013 & 0 & 0.3660 & URPSet $_{9}$ \\
\hline pms0001, pms $0005, p m s 0020$ & 0 & 0.3562 & URPSet $_{10}$ \\
\hline pms0001, pms0005, pms0006, pms0007 & 0 & 0.3497 & URPSet $_{11}$ \\
\hline pms0001, pms0004, pms0020 & 0 & 0.3122 & URPSet $_{12}$ \\
\hline pms0023, pms0024 & 0 & 0.3097 & URPSet $_{13}$ \\
\hline pms0006, pms0008 & 0 & 0.2975 & URPSet $_{14}$ \\
\hline pms0013, pms0031 & 0 & 0.2943 & URPSet $_{15}$ \\
\hline pms $0006, p m s 0036$ & 0 & 0.2869 & URPSet 16 \\
\hline pms0013, pms 0021 & 0 & 0.2804 & URPSet $_{17}$ \\
\hline pms 0007, pms 0036 & 0 & 0.2494 & URPSet 18 \\
\hline pms 0012, pms 0021 & 0 & 0.2405 & URPSet 19 \\
\hline pms 0013 , pms 0036 & 0 & 0.2380 & URPSet $_{20}$ \\
\hline pms0006, pms0012 & 0 & 0.2372 & URPSet $_{21}$ \\
\hline pms 0012, pms 0029 & 0 & 0.2282 & URPSet $_{22}$ \\
\hline pms 0012, pms 0013 & 0 & 0.2234 & URPSet $_{23}$ \\
\hline pms $0012, p m s 0036$ & 0 & 0.2119 & URPSet $_{24}$ \\
\hline pms0001, pms0004, pms0005, pms0007 & 0 & 0.2014 & URPSet $_{25}$ \\
\hline
\end{tabular}


In Table 5, we list the top 25 permission combinations where the first permission in the listed patterns belongs to the normal permissions category. The permission combinations from URPSet ${ }_{1}$ and URPSet were $_{2}$ both required by more than $60 \%$ of the malware. In fact, we found that the INTERNET permission ( $p m s 0001$ ) is frequently requested along with other permissions and their support value are relatively high. The permission combination, INTERNET and RECEIVE_BOOT_COMPLETED were present in 55\% of the malware dataset. Other such patterns involving the INTERNET permission are listed in Table 5 .

Table 6: Unique Required Permission Sets in Malware Dataset ( $A C$ CESS_FINE(COARSE)_LOCATION)

\begin{tabular}{l|cc|c}
\hline Permission Set & \multicolumn{2}{|c|}{ Support } & Permission Set \\
& Clean & Malware & ID \\
\hline pms0002, pms0005, pms0020 & 0 & 0.2690 & URPSet $_{26}$ \\
pms0002, pms0004, pms0020 & 0 & 0.2576 & URPSet $_{27}$ \\
pms0002, pms0005, pms0023 & 0 & 0.2307 & URPSet $_{28}$ \\
pms0002, pms0004, pms0023 & 0 & 0.2234 & URPSet $_{29}$ \\
\hline
\end{tabular}

Table 7: Unique Required Permission Sets in Malware Dataset (SMS)

\begin{tabular}{l|cc|c}
\hline Permission Set & \multicolumn{2}{|c|}{ Support } & Permission Set \\
& Clean & Malware & ID \\
\hline pms0030, pms0036 & 0 & 0.3228 & URPSet $_{30}$ \\
pms0021, pms0036 & 0 & 0.3163 & URPSet $_{31}$ \\
pms0031, pms0036 & 0 & 0.2690 & URPSet $_{32}$ \\
pms0029, pms0036 & 0 & 0.2674 & URPSet $_{33}$ \\
pms0021, pms0028 & 0 & 0.2519 & URPSet $_{34}$ \\
\hline
\end{tabular}

Table 8: Unique Required Permission Sets in Malware Dataset ( $C O N$ TACTS)

\begin{tabular}{l|cc|c}
\hline Permission Set & \multicolumn{2}{|c|}{ Support } & Permission Set \\
& Clean & Malware & ID \\
\hline pms0008, pms0030 & 0 & 0.3269 & URPSet $_{35}$ \\
pms0008, pms0021 & 0 & 0.2894 & URPSet $_{36}$ \\
pms0008, pms 0031 & 0 & 0.2649 & URPSet $_{37}$ \\
pms0008, pms0029 & 0 & 0.2429 & URPSet $_{38}$ \\
pms0028, pms0036 & 0 & 0.2413 & URPSet $_{39}$ \\
pms0008, pms0028 & 0 & 0.2282 & URPSet $_{40}$ \\
pms0008, pms0013 & 0 & 0.2250 & URPSet $_{41}$ \\
pms0028, pms0029 & 0 & 0.2128 & URPSet $_{42}$ \\
\hline
\end{tabular}


Table 9: Unique Required Permission Sets in Malware Dataset (WRITE_EXTERNAL_STORAGE)

\begin{tabular}{l|cc|c}
\hline Permission Set & \multicolumn{2}{|c|}{ Support } & Permission Set \\
& Clean & Malware & ID \\
\hline pms0004, pms0006, pms0023 & 0 & 0.4475 & URPSet $_{43}$ \\
pms0004, pms0030 & 0 & 0.3896 & URPSet $_{44}$ \\
pms $_{\text {p } 0004, p m s 0005, p m s 0020}$ & 0 & 0.3106 & URPSet $_{45}$ \\
pms0004, pms0021 & 0 & 0.2462 & URPSet $_{46}$ \\
pms $_{\text {p }} 0004$, pms0020, pms0023 & 0 & 0.2258 & URPSet $_{47}$ \\
pms0004, pms0029 & 0 & 0.2022 & URPSet $_{48}$ \\
\hline
\end{tabular}

Table 10: Unique Required Permission Sets in Malware Dataset (READ_PHONE_STATE)

\begin{tabular}{l|cc|c}
\hline Permission Set & \multicolumn{2}{|c|}{ Support } & Permission Set \\
& Clean & Malware & ID \\
\hline pms0005, pms0013 & 0 & 0.5453 & URPSet $_{49}$ \\
pms0005, pms0031 & 0 & 0.5094 & URPSet $_{50}$ \\
pms0005, pms0021 & 0 & 0.4190 & URPSet $_{51}$ \\
pms0005, pms0029 & 0 & 0.3798 & URPSet $_{52}$ \\
pms0005, pms0008 $_{\text {pms0005, pms0036 }}$ & 0 & 0.3538 & URPSet $_{53}$ \\
pms0005, pms0028 & 0 & 0.3350 & URPSet $_{54}$ \\
pms0005, pms0012 & 0 & 0.2934 & URPSet $_{55}$ \\
\hline
\end{tabular}


In Table 6- 10, we present the permission patterns that can have an impact on the following actions: access location information, read/write/send and receive SMS, access to contact list, write to external storage and access to phone state.

\subsubsection{Unique Used Permission (UUP) Patterns}

In Table 11, we list the combinations of the used permissions that are unique to the malware dataset only. It can be noted that the INTERNET permission is included in the top 3 permission combinations, UUPSet U $_{1}$ to $U_{U P S e t}$ and appears in over $40 \%$ of the malware samples. The combination of INTERNET and READ_PHONE_STATE permission is another frequent permission pattern, as depicted by UUPSet 21 and UUPSet 30 .

Table 11: Unique Used Permission Sets in Malware Dataset

\begin{tabular}{|c|c|c|c|}
\hline \multirow[t]{2}{*}{ Permission Set } & \multicolumn{2}{|c|}{ Support } & \multirow{2}{*}{$\begin{array}{l}\text { Permission Set } \\
\text { ID }\end{array}$} \\
\hline & Clean & Malware & \\
\hline pms0001, pms0005, pms0006, pms0007 & 0 & 0.5542 & UUPSet $_{1}$ \\
\hline pms0001, pms0005, pms0011 & 0 & 0.4687 & UUPSet $_{2}$ \\
\hline pms0001, pms0006, pms0011 & 0 & 0.4320 & UUPSet $_{3}$ \\
\hline pms0005, pms0006, pms0011 & 0 & 0.4312 & $\mathrm{UUPSet}_{4}$ \\
\hline pms0001, pms 0007, pms 0011 & 0 & 0.4149 & UUPSet $_{5}$ \\
\hline pms0005, pms 0007, pms 0011 & 0 & 0.4133 & UUPSet $_{6}$ \\
\hline pms0006, pms0007, pms0011 & 0 & 0.3855 & $\mathrm{UUPSet}_{7}$ \\
\hline pms0001, pms0002, pms0005, pms0007 & 0 & 0.3423 & UUPSet $_{8}$ \\
\hline pms0001, pms0021 & 0 & 0.3358 & UUPSet $_{9}$ \\
\hline pms0005, pms0021 & 0 & 0.3236 & UUPSet $_{10}$ \\
\hline pms0001, pms 0002, pms 0011 & 0 & 0.2845 & UUPSet $_{11}$ \\
\hline pms0002, pms0005, pms0011 & 0 & 0.2845 & UUPSet $_{12}$ \\
\hline pms0001, pms0002, pms0006, pms0007 & 0 & 0.2829 & UUPSet $_{13}$ \\
\hline pms0002, pms0005, pms0006, pms0007 & 0 & 0.2829 & UUPSet $_{14}$ \\
\hline pms0002, pms0006, pms0011 & 0 & 0.2755 & UUPSet $_{15}$ \\
\hline pms0007, pms0021 & 0 & 0.2723 & UUPSet $_{16}$ \\
\hline pms0001, pms0020 & 0 & 0.2600 & UUPSet $_{17}$ \\
\hline pms0005, pms0020 & 0 & 0.2600 & UUPSet $_{18}$ \\
\hline pms0002, pms0007, pms0011 & 0 & 0.2568 & UUPSet $_{19}$ \\
\hline pms0006, pms 0020 & 0 & 0.2511 & UUPSet $_{20}$ \\
\hline pms0001, pms 0005, pms 0010 & 0 & 0.2421 & UUPSet $_{21}$ \\
\hline pms0001, pms0007, pms0010 & 0 & 0.2413 & UUPSet $_{22}$ \\
\hline pms0005, pms0007, pms 0010 & 0 & 0.2413 & UUPSet $_{23}$ \\
\hline pms0011, pms0020 & 0 & 0.2380 & UUPSet $_{24}$ \\
\hline pms0001, pms0006, pms0010 & 0 & 0.2372 & UUPSet $_{25}$ \\
\hline pms0005, pms0006, pms0010 & 0 & 0.2372 & UUPSet $_{26}$ \\
\hline pms0006, pms 0007, pms 0010 & 0 & 0.2364 & $\mathrm{UUPSet}_{27}$ \\
\hline pms0006, pms0021 & 0 & 0.2348 & UUPSet $_{28}$ \\
\hline pms 0007, pms 0020 & 0 & 0.2266 & UUPSet $_{29}$ \\
\hline pms0001, pms0003, pms0005, pms0007 & 0 & 0.2258 & UUPSet 30 \\
\hline pms0002, pms0020 & 0 & 0.2185 & UUPSet $_{31}$ \\
\hline
\end{tabular}

Interestingly, READ_LOGS (pms0011) permission appears in one-third of 
the permission patterns presented in Table 11. It is often combined with the INTERNET (pms0001) and ACCESS_FINE_LOCATION ( pms0002) permissions. The remaining patterns include combinations of network-related and SMS permissions.

\subsubsection{Common Required Permission (CRP) Patterns}

Previously, we presented the permission patterns that were unique to malicious applications only. In Table 12 , we list the permission combinations that appear in both clean and malware datasets. However, it can be observed based on the support value difference that the permission patterns are more prevalent in the malware dataset, as shown by the negative support difference values.

Table 12: Common Required Permission Sets in Both Clean and Malware Datasets

\begin{tabular}{|c|c|c|c|c|}
\hline \multirow[t]{2}{*}{ Permission Set } & \multicolumn{2}{|c|}{ Support } & \multirow[t]{2}{*}{ Difference } & \multirow{2}{*}{$\begin{array}{l}\text { Permission Set } \\
\text { ID }\end{array}$} \\
\hline & Clean & Malware & & \\
\hline pms0001, pms0005 & 0.3121 & 0.9307 & -0.6186 & CRPSet $_{1}$ \\
\hline pms0005 & 0.3187 & 0.9340 & -0.6153 & $\mathrm{CRPSet}_{2}$ \\
\hline pms0005, pms0023 & 0.0236 & 0.6308 & -0.6072 & $\mathrm{CRPSet}_{3}$ \\
\hline pms0030 & 0.0147 & 0.6210 & -0.6064 & $\mathrm{CRPSet}_{4}$ \\
\hline pms 0001, pms 0023 & 0.0505 & 0.6349 & -0.5844 & $\mathrm{CRPSet}_{5}$ \\
\hline pms 0023 & 0.0522 & 0.6349 & -0.5827 & $\mathrm{CRPSet}_{6}$ \\
\hline pms0006, pms0023 & 0.0399 & 0.6031 & -0.5632 & $\mathrm{CRPSet}_{7}$ \\
\hline pms0005, pms0006 & 0.2421 & 0.7905 & -0.5485 & $\mathrm{CRPSet}_{8}$ \\
\hline pms0001, pms $0005, p m s 0006$ & 0.2421 & 0.7897 & -0.5477 & CRPSet $_{9}$ \\
\hline pms0001, pms0004, pms0005 & 0.1328 & 0.6544 & -0.5216 & CRPSet $_{10}$ \\
\hline pms0004, pms0005 & 0.1337 & 0.6553 & -0.5216 & CRPSet $_{11}$ \\
\hline pms 0013 & 0.0489 & 0.5542 & -0.5053 & CRPSet $_{12}$ \\
\hline pms 0031 & 0.0106 & 0.5159 & -0.5053 & CRPSet $_{13}$ \\
\hline pms0001, pms0004, pms0005, pms0006 & 0.1149 & 0.5623 & -0.4474 & CRPSet $_{14}$ \\
\hline pms0004, pms0005, pms0006 & 0.1149 & 0.5623 & -0.4474 & CRPSet $_{15}$ \\
\hline pms0004, pms0023 & 0.0293 & 0.4637 & -0.4344 & CRPSet $_{16}$ \\
\hline pms0021 & 0.0277 & 0.4417 & -0.4140 & CRPSet $_{17}$ \\
\hline
\end{tabular}

Permissions, such as INTERNET ( $p m s 0001)$, READ_PHONE_STATE ( $p m s 0005)$, ACCESS_NETWORK_STATE $(p m s 0006)$ and ACCESS_WIFI_STATE ( $p m s 0023)$, are present in different combinations and appear in more than $40 \%$ of the malware dataset. One interesting observation is $C R P$ Set $_{14}$ which comprises of a combination of four permissions and appear in a significant $40 \%$ of the malicious applications.

\subsubsection{Common Used Permission (CUP) Patterns}

In Table 13, we present the used permission combinations that appeared in both the clean and malware datasets. We note that although both 
datasets have the same permission patterns, the ones in the malware dataset have higher support values.

In such patterns, only five permissions are found: INTERNET ( $p m s 0001)$, READ_PHONE_STATE (pms0005), ACCESS_NETWORK_STATE ( $p m s 0006)$, VIBRATE (pms0007) and READ_LOGS ( pms0011). It is also worth noting that CUPSet and $C U P S e t_{2}$ have almost the same support difference, hence indicating that the occurrence of these permission combinations are highly relevant. Moreover, we observed that even though READ_LOGS (pms0011) permission did not appear in the common required permission patterns, but it appeared in three common unique permission patterns READ_LOGS, CUPSet - CUPSet9.

Table 13: Common Used Permission Sets in Both Clean and Malware Datasets

\begin{tabular}{l|cc|c|c}
\hline Permission Set & \multicolumn{2}{|c|}{ Support } & Difference & Permission Set \\
& Clean & Malware & & \\
\hline pms0001, pms0005 & 0.2991 & 0.9152 & -0.6161 & CUPSet $_{1}$ \\
pms0005 & 0.3032 & 0.9169 & -0.6137 & CUPSet $_{2}$ \\
pms0001, pms 0005, pms 0006 & 0.2363 & 0.7718 & -0.5355 & CUPSet $_{3}$ \\
pms0005, pms0006 & 0.2363 & 0.7718 & -0.5355 & CUPSet $_{4}$ \\
pms0001, pms0005, pms 0007 & 0.2168 & 0.6512 & -0.4344 & CUPSet $_{5}$ \\
pms0005, pms0007 & 0.2192 & 0.6528 & -0.4336 & CUPSet $_{6}$ \\
pms0005, pms0011 & 0.0538 & 0.4686 & -0.4148 & CUPSet $_{7}$ \\
pms0011 & 0.0693 & 0.4760 & -0.4067 & CUPSet $_{8}$ \\
pms0001, pms0011 & 0.0685 & 0.4711 & -0.4026 & CUPSet $_{9}$ \\
\hline
\end{tabular}

\subsection{Discussion}

The Android smartphone has gained in popularity in the past few years. Two main factors that contributed towards this change is the open-source nature of the platform and the flexibility provided to users and developers alike when downloading and developing Android applications, respectively. However, not all applications present on the application markets, both official and third-party, are clean. Previous work showed that malware authors take advantage of the Android permission system to entice users into installing unsafe applications. As such, this study aims to understand required and used permissions by Android applications by applying data mining techniques to find emerging permission patterns that can be used to contrast clean and malicious applications. 


\subsubsection{Observations from Statistical Analysis}

Our proposed methodology considers the patterns of both required and used permissions. From our statistical analysis in Section 3.1, we observe that the INTERNET permission remain the most required (97.72\%) and used $(94.62 \%)$ permission in our experimental dataset. We also find, from Tables 1 and 2 , that there is a significant difference in the frequencies of required and used permissions for the clean and malware datasets. This observation aligns with the one made by Felt et al. in [34] and therefore, demonstrates that both clean and malicious applications can be over-privileged. Till date, most of the proposed solutions have only considered required permissions which are extracted from the AndroidManifest.xml files. From our statistical results, we argue that used permissions should also be considered as part of the feature set and as such, can aid towards malicious application detection.

Additionally, in order to have a comparative distribution of required and used permissions, we extend the statistical analysis by applying the biclustering algorithm to generate visualization maps. It should be noted that we apply biclustering mainly to visualize the distribution of required and used permissions. Thus, we do not aim to identify clusters of permissions. As expected, since applications generally request more permissions than actually used, the distribution of required permissions for clean and malware datasets is more sparsed than that of used permissions - as shown in Figures 2 and 3 , respectively. The visualization maps provide researchers and analysts alike with a first-hand overview of permissions that are common and unique between clean and malicious applications. Furthermore, the maps can be used as a substitution for statistical analysis as it is a time-consuming process and requires little or no margin of error. As Zhou et al. pointed out in [45, the increasing number of malicious applications is mostly due to how easy it is to produce repackaged applications. These applications can contain additional advertising libraries, malicious code and most importantly, additional permissions that were previously not present in the original applications. The maps can outline these differences in permissions for clean and malicious applications. Subsequently, the permission distribution visuals can also portray required and used permissions for different variants belonging to the same malware family. Hence, the maps can be used for a preliminary analysis of zero-day samples detected by antivirus companies.

\subsubsection{Analysis of Permission Visualizations}

From the biclustering results, we observed that several applications, clean or malicious, have more than one (required and used) permission in 
common between them. Conversely, we also noticed similar observation for unique required and used permissions for the two datasets. In general, existing work [12], [30], 33] consider only individual permissions when studying permission request patterns. Therefore, we put forward a method that consider co-dependencies between permissions that are unique and common amongst clean and malicious applications. In our paper, we apply a data mining technique known as contrast mining to generate permission sets that constitute of multiple permissions and can be used to reinforce similarities and contrasts between clean and malicious applications.

From our findings, we observe that 23 permissions (as shown in Table 4), out of a total of 128 permissions that were extracted from our dataset, appear frequently in the permission sets. It is also worth noting that two of the Dangerous permissions, WRITE_EXTERNAL_STORAGE (pms0004) and READ_PHONE_STATE $(p m s 0005)$ can be implicitly granted to an application that utilizes API level 3 or lower, as described in [27. This implies that if these two permissions are not recorded as required permissions, they can still be present as used permissions. Upon further investigation, we found that whilst the number of required permission for WRITE_EXTERNAL_STORAGE exceeds that of used permission, the same observation cannot be made for READ_PHONE_STATE. From Tables 1 and 2, it can be noticed that the number of clean applications (391) which required READ_PHONE_STATE is lesser than the number of clean applications (457) that used this permission. Although we do not keep record of the API level information for the applications in our dataset; however, in this case, we can deduce that some clean applications from the set of 457 applications were implicitly granted the READ_PHONE_STATE permission. This permission can have nefarious ramifications on users' private information as it allows an application to read unique device identifiers such as, International Mobile Equipment Identity (IMEI), International Mobile Subscriber Identity (IMSI) and the SIM serial number, as shown by [46].

\subsubsection{Analysis of Contrast Permission Patterns}

In Section 4.2, we present the most significant permission sets generated by contrast mining. Based on our experimental results, we found that a large number of required and used permission sets were unique in malicious applications only. This is a good indication that the permission sets can be further applied during the malware detection phase to identify malicious applications. For normal required permissions, we observed from Table 5 that

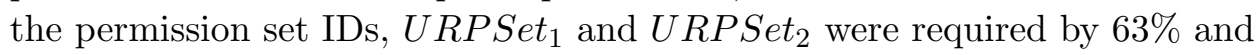
$60 \%$ of the malicious applications in our dataset, respectively. We deduce 
that this might be the case due to the fact that $25 \%$ of our experimental malware samples (malicious applications) belong to the DroidKungFu3 malware family. As demonstrated in [47], malware samples classified under DroidKungFu3 attempt to extract device ID, network-related information and send all information back to the attacker's server.

As for the Dangerous required permissions sets included in Tables 6 to 10, we notice several interesting permission sets on which we provide further explanation. For permission set IDs URPSet 26 and URPSet 27 , we find that $25 \%$ of malicious applications require both ACCESS_FINE_LOCATION (pms0002) and ACCESS_COARSE_LOCATION ( $p m s 0020)$ permissions. While ACCESS_COARSE_LOCATION grants access to GPS location sources, ACCESS_COARSE_LOCATION is used for location information related to network sources. However, the documentation [48] provided by Google specifies that if a developer requires network and GPS location information, they do not need to include both permissions in the application; only requesting ACCESS_FINE_LOCATION should suffice. The presence of unused permission can be exploited via permission inheritance during inter-component communications, as explained in [49].

Moreover, we observe that for SMS-related permissions: SEND_SMS (pms0021), RECEIVE_SMS ( $p m s 0029)$, READ_SMS ( $p m s 0030)$ and WRITE_SMS ( $p m s 0031)$, as shown in Table 7, the CALL_PHONE (pms0036) permission is associated with these four permissions in over $25 \%$ of the malicious applications in our dataset. The CALL_PHONE permission allows an application to proceed with making a phone call without going through the usual dialer interface. Some malware samples exploit the aforementioned permission combinations to make premium calls and send text messages to premium numbers.

As for the used permission sets that are unique in our malware dataset (Table 11), we observed that the permission set: INTERNET (pms0001), READ_PHONE_STATE $(p m s 0005)$, ACCESS_NETWORK_STATE $(p m s 0006)$, VIBRATE ( $p m s 0007$ ) with permission set ID UUPSet I $_{1}$ was used by $55 \%$ of the malware samples. Interestingly, we also found that the same permission set was present in Table 5 under the permission set ID URPSet ${ }_{11}$, with the only exception that it was required by only $35 \%$ of the malware samples. We attribute this $20 \%$ difference to the observation made in Section 4.3.2, on the READ_PHONE_STATE permission. Moreover, it can be noted from Table 11 that the READ_LOGS ( $p m s 0011$ ) permission is frequently associated with the permission sets and appeared in $25 \%$ to $50 \%$ of the malware dataset. There was previously no indication that (pms0011) was a highly used permission among malicious applications as the permission did not appear in the Top 20 most used permission, in Table 2. This further consolidates our argument that permission patterns cannot be generated by only considering the 
number of frequencies for that particular permission.

Furthermore, we also noted that there are several permission sets which appeared in both clean and malware datasets, shown in Tables 12 and 13 . The negative support difference given in the table shows that the permission sets are more prevalent in malicious applications than in clean ones. We observed that the top two permission sets, CRPSet ${ }_{1}$ and $C R P$ Set $_{2}$ in Table 12 and $C U P$ Set $_{1}$ and $C U P$ Set $_{11}$ in Table 13 are the same. However, we noted some discrepancies for permissions such as READ_LOGS (pms0011) and VIBRATE ( $p m s 0007$ ). Similar to our previous observations, it appears that the above two permissions are not recorded during the generation of required permission sets but for used permission sets, their high support values indicate that they are highly significant.

\section{Conclusion}

In this paper, we studied the Android permission system as the smartphone platform makes use of permissions to regulate access to system resources and users' private information. In order to understand and identify permission patterns, the existing work consider only those permissions that are declared in the AndroidManifest.xml files. We refer to those permissions as required permissions. However, there is another permission check that takes place after an application has been installed and is executed by the smartphone OS. We refer to such permissions as used permissions.

In our work, we considered the implications of incorporating used permissions in permission patterns and determined their usefulness in contrasting between clean and malicious applications. We proposed an efficient pattern mining method to generate a set of emerging contrast permission patterns for our clean and malware dataset. Based on our experimental results, we observed that there are several permissions that do not appear in the required permission sets but are present in the used permission sets. We found out that there is a discrepancy in the official documentation that allows application with API level 3 or level to implicitly inherit certain permissions although they are not declared in the AndroidManifest.xml file.

Additionally, the patterns obtained from our proposed methodology ensures that the permission sets were not generated by chance as we used support values to measure and rank the patterns. This is an improvement over Frank et al.'s work [12] where the authors had to simulate permission request data to test their generated patterns. Last but not least, since obfuscation methods cannot be applied to Android permissions, the generated

permission sets can be used to contrast clean and malicious applications. In 
the future, we would like to work on finding contrasting patterns that can differentiate between an original application and a repackaged one.

\section{References}

[1] PC Magazine. Encyclopedia, Accessed in December 2012. http: //www.pcmag.com/encyclopedia_term/0, 2542, $t=$ Smartphone\& $i=$ $51537,00 . \mathrm{asp}$.

[2] Google. Nexus, Accessed in March 2013. http://www.google.com/ nexus.

[3] Apple Inc. iphone, Accessed in March 2013. http://www.apple.com/ iphone.

[4] Research in Motion Ltd. Blackberry, Accessed in March 2013. http: //au.blackberry.com.

[5] Microsoft. Windows phone, Accessed in March 2013. http://www. windowsphone.com/en-gb.

[6] Apple Inc. Welcome to apple store, Accessed in March 2013. http: //store.apple.com/au.

[7] BlackBerry. Blackberry world, Accessed in March 2013. http:// appworld.blackberry.com/webstore.

[8] Google. Google play, Accessed in March 2013. https://play.google. com/store.

[9] P. Gilbert and C. Byung-Gon and P. C. Landon and J. Jaeyeon. Vision: automated security validation of mobile apps at app markets. In Proceedings of the 2nd International Workshop on Mobile Cloud Computing and Services (MCS 2011), pages 21-26, Washington, USA, June 2011.

[10] Google. Malware - what's the policy?, Accessed in March 2013. http://support.google.com/adwordspolicy/bin/answer $\cdot$ py?hl= en\&answer=1308246\&topic=1626336\&path=1316546\&ctx=leftnav.

[11] Y. Zhou and X. Jiang. Dissecting Android malware: Characterization and evolution. In Proceedings of the IEEE Symposium on Security and Privacy (SP 2012), pages 95-109, San Francisco, CA, May 2012. 
[12] M. Frank, B. Dong, A.P. Felt, and D. Song. Mining permission request patterns from Android andFacebook applications. In Proceedings of the IEEE International Conference on Data Mining (ICDM 2012), pages 1-16, Brussels, Belgium, December 2012.

[13] D. Barrera, H.G. Kayacik, P.C. van Oorschot, and A. Somayaji. A methodology for empirical analysis of permission-based security models and its application to Android. In Proceedings of the 17th ACM conference on Computer and communications security (CCS 2010), pages 73-84, Chicago, Illinois, USA, October 2010.

[14] A.P. Felt, K. Greenwood, and D. Wagner. The effectiveness of application permissions. In Proceedings of the USENIX Conference on Web Application Development (WebApps 2011), pages 1-12, Portland, Oregon, June 2011.

[15] A.P. Felt, E. Ha, S. Egelman, A. Haney, E. Chin, and D. Wagner. Android permissions: User attention, comprehension and behavior. In Proceedings of the Symposium on Usable Privacy and Security (SOUPS 2012), number 3, pages 1-14, Washington, D.C., July 2012.

[16] P.H. Chia, Y. Yamamoto, and N. Asokan. Is this app safe? a large scale study on application permissions and risk signals. In Proceedings of the 21st international conference on World Wide Web (WWW 2012), pages 311-320, Lyon, France, April 2012.

[17] International Secure Systems Lab. Andrubis: Analyzing Android binaries, Accessed in May 2012. http://anubis.iseclab.org/?action= home.

[18] virusTotal. Credits \& acknowlegements, Accessed in March 2013. https://www.virustotal.com/en/about/credits.

[19] Open Handset Alliance. Android, Accessed in February 2013. http: //www.openhandsetalliance.com/android_overview.html.

[20] F. Ableson. Introduction to Android development, Accessed in January 2013. http://www.ibm.com/developerworks/library/ os-android-devel.

[21] Google. Android SDK, Accessed in January 2013. http://developer. android.com/sdk/index.html. 
[22] The Eclipse Foundation. Eclipse ide for java developers, Accessed in March 2013. http://www.eclipse.org/downloads/packages/ eclipse-ide-java-developers/junosr1.

[23] Android Open Source Project. Bytecode for the dalvik virtual machine, Accessed in January 2013. http://source.android.com/tech/ dalvik/dalvik-bytecode.html.

[24] Google. Google play, Accessed in March 2013. https://play.google. com.

[25] H. Lockheimer. Android and Security, Accessed in March 2013. http://googlemobile.blogspot.com.au/2012/02/ android-and-security.html.

[26] J.R. Raphael. Inside Android 4.2's powerful new security system, Accessed in November 2012. http://blogs.computerworld.com/ android/21259/android-42-security.

[27] Google. Android permissions, Accessed in December 2012. http://developer.android.com/guide/topics/manifest/ permission-element.html.

[28] A. Bartel, J. Klein, M. Monperrus, and Y.L. Traon. Automatically securing permission-based software by reducing theattack surface - an application to Android. In Proceedings of the 27th IEEE/ACM International Conference on Automated Software Engineering (ASE 2012), pages 274-277, Essen, Germany, September 2012.

[29] B. Sanz, I. Santos, C. Laorden, X. Ugarte-Pedrero, P.G. Bringas, and G. Álvarez. PUMA: Permission usage to detect malware in Android. In Proceedings of the International Joint Conference CISIS'12ICEUTE'12-SOCO'12 Special Sessions, volume 189 of Advances in Intelligent Systems and Computing, pages 289-298. Springer Berlin Heidelberg, September 2013.

[30] I. Rassameeroj and Y. Tanahashi. Various approaches in analyzing Android applications with its permission-based security models. In Proceedings of the IEEE International Conference on Electro/Information Technology (EIT 2011), number 44, pages 1-6, Minnesota, USA, May 2011. 
[31] J. Sahs and L. Khan. A machine learning approach to Android malware detection. In Proceedings of the European Intelligence and Security Informatics Conference (EISIC 2012), pages 141-147, Odense, Denmark, August 2012.

[32] D.J. Wu, C.H. Mao, T.E. Wei, H.M. Lee, and K.P. Wu. DroidMat: Android malware detection through manifest and API calls tracing. In Proceedings of the 2012 Seventh Asia Joint Conference on InformationSecurity (Asia JCIS 2012), pages 62-69, Tokyo, Japan, August 2012.

[33] Y. Zhou, Z. Wang, W. Zhou, and X. Jiang. Hey, You, Get Off of My Market: Detecting Malicious Appsin Official and Alternative Android Markets. In In Proceedings of the 19th Annual Network and Distributed System Security Symposium (NDSS 2012), pages 1-13, San Diego, California, February 2012.

[34] A.P. Felt, E. Chin, S. Hanna, D. Song, and D. Wagner. Android permissions demystified. In Proceedings of the ACM Conference and Communications Security (CCS 2011), pages 627-638, Chicago, U.S.A, October 2011.

[35] T. Vidas, N. Christin, and L. Cranor. Curbing Android permission creep. In Proceedings of the 2011 Web 2.0 Security and Privacy Workshop (W2SP 2011), pages 1-5, Oakland, CA, May 2011.

[36] A.P. Felt, M. Finifter, E. Chin, S. Hanna, and D. Wagner. A survey of mobile malware in the wild. In Proceedings of the ACM Workshop on Security and Privacy in Mobile Devices (SPSM 2011), pages 3-14, Chicago, U.S.A, October 2011.

[37] S.C. Madeira and A.L. Oliveira. Biclustering algorithms for biological data analysis: A survey. IEEE Transactions on computational Biology and Bioinformatics, 1(1):24-45, 2004.

[38] G.J. Szekely and M.L. Rizzo. Hierarchical clustering via joint betweenwithin distances: Extending ward's minimum variance method. Journal of Classification, 22(2):151-183, 2005.

[39] A. Fernández and S. Gómez. Solving non-uniqueness in agglomerative hierarchical clustering using multidendrograms. Journal of Classification, 25(1):43-65, 2008.

[40] Joe H. Ward. Hierarchical grouping to optimize an objective function. Journal of The American Statistical Association, 58:236-244, 1963. 
[41] R. Agrawal, T. Imieinski, and A. Swami. Mining association rules between sets of items in large databases. In P. Buneman and S. Jajodia, editors, Proceedings of the ACM SIGMOD International Conference on the Management of Data (COMAD 1993), pages 207-216, Washington DC, 1993. ACM Press.

[42] S. Liu, R. Law, J. Rong, G. Li, and J. Hall. Analyzing changes in hotel customers' expectations by trip mode. International Journal of Hospitality Management, 34:359-371, 2013.

[43] J. Rong, H.Q. Vu, R. Law, and G. Li. A behavioral analysis of web sharers and browsers inhong kong using targeted association rule mining. Tourism Management, 33(4):731-740, 2012.

[44] R. Law, R. Rong, H.Q. Vu, G. Li, and H.A Lee. Identifying changes and trends in hong kong outbound tourism. Tourism Management, 32(5):1106-1114, 2011.

[45] W. Zhou, Y. Zhou, X. Jiang, and P. Ning. Detecting repackaged smartphone applications in third-party Android marketplaces. In Proceedings of the second ACM conference on Data and ApplicationSecurity and Privacy (CODASPY 2012), pages 317-326, San Antonio, Texas, USA, February 2012.

[46] V. Moonsamy, M. Alazab, and L. Batten. Towards an understanding of the impact of advertising on data leaks. International Journal of Security and Networks, 7(3):181-193, 2012.

[47] F-Secure. Trojan:android/droidkungfu.c, Accessed in January 2013. http://www.f-secure.com/v-descs/trojan_android_ droidkungfu_c.shtml.

[48] Android Developer. Location strategies, Accessed in January 2013. http://developer.android.com/guide/topics/location/ strategies.html.

[49] E. Chin, A.P. Felt, K. Greenwood, and D. Wagner. Analyzing interapplication communication in Android. In Proceedings of the 9th Annual International Conference on Mobile Systems, Applications, and Services (MobiSys 2011), pages 239-252, Washington, USA, June 2011. 